F. Halter-Koch

Nagoya Math. J.

Vol. 120 (1990), 61-66

\title{
A NOTE ON RAY CLASS FIELDS OF GLOBAL FIELDS
}

\author{
FRANZ HALTER-KOCH
}

\section{$\S 1$. Introduction}

The notion of a ray class field, which is fundamental in Takagi's class field theory, has no immediate analogon in the function field case. The reason for this lies in the lacking of a distinguished maximal order. In this paper I overcome this difficulty by a relative version of the notion of ray class fields to be defined for every holomorphy ring of the field. The prototype for this new notion is M. Rosen's definition of a Hilbert class field for function fields [6].

The construction applies for both number and function fields and includes the "classical" ray class fields in the number field case. Finally, as in the classical case, Artin's reciprocity law together with Cebotarev's Theorem, yields a Dirichlet Theorem for arithmetical progressions in holomorphy rings.

\section{§ 2. Notations; Class groups}

A global field is either an algebraic number field of finite degree over $\mathbf{Q}$ or an algebraic function field in one variable over a finite field. I shall use class field theory in its idelic version as derived in [1], [4] or [8].

In connection with a global field $K$ I shall use the following notations:

$S(K)$ denotes the set of all non-archimedian places of $K$; every $v \in$ $S(K)$ will be identified with the associated normalized valuation $v: K \rightarrow$ $\mathrm{Z} \cup\{\infty\}$. In the function field case $\operatorname{deg}(v)$ denotes the degree of $v$.

$\bar{S}(K)$ denotes the set of all places, and $S_{\infty}(K)$ denotes the set of all real places of $K$. In the function field case $S(K)=\bar{S}(K)$ and $S_{\infty}(K)=\varnothing$.

For $v \in \bar{S}(K)$ let $K_{v}$ be the completion of $K$ at $v$; for $v \in S(K)$ let $R_{v}$ be the valuation ring, $P_{v}$ the valuation ideal and $U_{v}=R_{v} \backslash P_{v}$ the unit group at $v$. Let $\mathscr{D}_{K}$ be the group of divisors and $\mathscr{C}_{K}$ the divisor class group of $K$. I view $\mathscr{D}_{K}$ as the free abelian group on $S(K)$, written addi-

Received February 5, 1990. 
tively; for $D \in \mathscr{D}_{K}$, let $\operatorname{supp}(D)=\{v \in S(K) \mid v(D) \neq 0\}$ be the support of $D$ and $[D] \in \mathscr{C}_{K}$ the divisor class containing $D$. In the function field case write deg for the degree of a divisor or a divisor class, and denote by $\dot{\mathscr{D}}_{K}$ resp. $\dot{\mathscr{C}}_{K}$ the group of all divisors resp. divisor classes of degree 0 .

For a finite subset $S \subset S(K), S \neq \varnothing$ in the function field case, define

$$
R_{S}=\bigcup_{v \in S(K) \backslash S}\left(R_{v} \cap K\right) \text {; }
$$

$R_{S}$ is called the holomorphiy ring of $S . R_{S}$ is a Dedekind ring with quotient field $K$; for $v \in S(K) \backslash S$ set

$$
P_{v, S}=P_{v} \cap R_{S} .
$$

Then $\left\{P_{v, S} \mid v \in S(K) \backslash S\right\}$ is the set of all non-zero prime ideals of $R_{S}$; proofs of these facts may be found in $[9 ;$ ch. 4] for the number field case and in $[3 ;$ ch. 2.7] for the function field case.

Let $\mathscr{I}_{S}$ be the group of fractional ideals and $\mathscr{C}_{S}$ the ideal class group of $R_{S}$; for $\mathbf{c} \in \mathscr{I}_{S}$ let $[\mathbf{c}] \in \mathscr{C}_{S}$ be the class containing c. Consider the epimorphism $i_{S}: \mathscr{D}_{K} \rightarrow \mathscr{C}_{S}$, defined by

$$
i_{S}\left(\sum_{v \in S(K)} n_{v} \cdot v\right)=\left[\prod_{v \in S(K) \backslash S} P_{v, S}^{n_{v}}\right] ;
$$

it induces an epimorphism

$$
\iota_{S}: \mathscr{C}_{K} \longrightarrow \mathscr{C}_{S}
$$

which has the following properties (see also [7; Prop. 1]):

LEMMA 1.

i) $\operatorname{ker}\left(\iota_{S}\right)=\left\{[D] \mid D \in \mathscr{D}_{K}\right.$, $\left.\operatorname{supp}(D) \subset S\right\}$.

ii) In the function field case,

$$
\left(\mathscr{C}_{S}: \iota_{S}\left(\dot{\mathscr{C}}_{K}\right)\right)=\operatorname{gcd}\{\operatorname{deg}(v) \mid v \in S\} .
$$

In particular, $\mathscr{C}_{s}$ is a finite group.

Proof. i) is obvious. To prove ii), observe that

$$
\operatorname{deg}\left(\operatorname{ker}\left(\iota_{s}\right)\right)=\delta \mathbf{Z}
$$

with $\delta=\operatorname{gcd}\{\operatorname{deg}(v) \mid v \in S\}$ and that deg induces an epimorphism $\operatorname{deg}^{*}: \mathscr{C}_{S}$ $\rightarrow \mathbf{Z} / \delta \mathbf{Z}$ with $\operatorname{ker}\left(\operatorname{deg}^{*}\right)=\iota_{S}\left(\dot{\mathscr{C}}_{K}\right)$.

Next I introduce $S$-ray class groups both in the idelic and the idealtheoretic version. Let $R_{S}$ be a holomorphy ring in a global field $K$ as above. A cycle of $R_{S}$ is a formal product 


$$
\mathscr{F}=\mathscr{F}_{0} \cdot w_{1} \cdot \cdots \cdot w_{m},
$$

where $m \geq 0, w_{1}, \cdots, w_{m} \in S_{\infty}(K)$, and

$$
\mathscr{F}_{0}=\prod_{v \in S(K) \backslash S} P_{v, S}^{v(\mathscr{F})}
$$

is an integral ideal of $R_{S}$ (we have $v(\mathscr{F}) \geq 0$ for all $v \in S(K) \backslash S$ and $v(\mathscr{F})=0$ for almost all $v$ ); set $S_{0}(\mathscr{F})=\{v \in S(K) \backslash S \mid v(\mathscr{F})>0\}$ and $S(\mathscr{F})$ $=S_{0}(\mathscr{F}) \cup\left\{w_{1}, \cdots, w_{m}\right\}$.

Let $J_{K}$ be the idele group of $K$; write $\alpha \in J_{K}$ in the form $\alpha=\left(\alpha_{v}\right)_{v \in \bar{S}(K)}$ and view $K^{\times}$as a subgroup of $J_{K}$ in the usual way. If $\mathscr{F}$ is a cycle of $R_{S}$ as above, put

$$
J_{K}^{(\mathscr{F})}=\left\{\alpha \in J_{K} \mid \alpha_{v}=1 \text { for all } v \in S(\mathscr{F})\right\}
$$

and

$$
J_{K, S}^{\mathscr{s}}=\left\{\alpha \in J_{K} \mid \alpha \equiv 1 \bmod \mathscr{F}, \alpha_{v} \in U_{v} \text { for all } v \in S(K) \backslash S\right\},
$$

where $\alpha \equiv 1 \bmod \mathscr{F}$ means $\alpha_{v} \in 1+P_{v}^{v(\mathscr{F})}$ for all $v \in S_{0}(\mathscr{F})$ and $\alpha_{w}>0$ for all $w \in\left\{w_{1}, \cdots, w_{m}\right\}$. The group $J_{K, S}^{\mathscr{q}} \cdot K^{\times} / K^{\times}$is an open subgroup of finite index in the idele class group $J_{K} / K^{\times}$(here I use $S \neq \varnothing$ in the function field case); the factor group $J_{K} / J_{K, S}^{s_{S}} \cdot K^{\times}$is called the idelic $S$-ray class group modulo $\mathscr{F}$.

Let $\mathscr{I}_{S}^{(\mathscr{F})}$ be the group of all fractional ideals $\mathbf{c} \in \mathscr{I}_{S}$ satisfying $v(\mathbf{c})$ $=0$ for all $v \in S_{0}(\mathscr{F})$, and

$$
\mathscr{S}_{S}^{(\mathscr{F})}=\left\{a R_{S} \mid a \in K^{\times}, a \equiv 1 \bmod \mathscr{F}\right\} \subset \mathscr{I}_{S}^{(\mathscr{F})} ;
$$

the quotient group $\mathscr{I}_{S}^{(*)} / \mathscr{S}_{S}^{(\mathscr{S})}$ is called the $S$-ray class group modulo $\mathscr{F}$. Let $E_{S}(\mathscr{F})$ be the set of all congruence classes $(a \bmod \mathscr{F})$ with $a \in R_{S}$ and $\left(a, \mathscr{F}_{0}\right)=1$ modulo those represented by units of $R_{S}$; then $E_{S}(\mathscr{F})$ is a finite group, and there is a canonical exact sequence

$$
1 \longrightarrow E_{S}(\mathscr{F}) \longrightarrow \mathscr{I}_{S}^{(\mathscr{F})} \mid \mathscr{S}_{S}^{(\mathscr{F})} \longrightarrow \mathscr{C}_{S} \longrightarrow 1,
$$

which shows that $\mathscr{I}_{S}^{(*)} / \mathscr{S}_{S}^{(*)}$ is a finite group and gives rise to a formula for its order.

For $\alpha \in J_{K}^{(\mathscr{g})}$ define

$$
\iota_{S}(\alpha)=\prod_{v \in S(K) \backslash S} P_{v, S}^{v\left(\alpha_{v}\right)} \in \mathscr{I}_{S}^{(\mathscr{F})} ;
$$

$\iota_{S}: J_{K}^{(\mathscr{F})} \rightarrow \mathscr{I}_{S}^{(\xi)}$ is a group epimorphism. Using the approximation theorem. it is easy to prove the following isomorphism statements. 
LEMMA 2.

i) The inclusion map $J_{K}^{(\mathscr{F})} \hookrightarrow J_{K}$ induces an isomorphism

$$
J_{K}^{(s)} / J_{K}^{(s)} \cap K^{\times} \cdot J_{K, S}^{\mathscr{s}} \stackrel{\sim}{\longrightarrow} J_{K} / K^{\times} \cdot J_{K, S}^{\mathscr{s}} \cdot
$$

ii) $\iota_{s}$ induces an isomorphism

$$
\bar{\iota}_{S}: J_{K}^{(s)} / J_{K}^{(s)} \cap K^{\times} \cdot J_{K, S}^{s^{(s)}} \longrightarrow \mathscr{I}_{S}^{(\mathscr{F})} / \mathscr{S}_{S}^{(\mathscr{F})} .
$$

\section{§ 3. Generalized ray class fields}

Let $K$ be a global field, $S \subset S(K)$ a finite set of places, $S \neq \varnothing$ in the function field case, and $\mathscr{F}$ a cycle of $R_{s}$. The abelian extension of $K$ belonging to the open subgroup $K^{\times} \cdot J_{K, S}^{\mathscr{\alpha}} / K^{\times}$of $J_{K} / K^{\times}$is called the $S$-ray class field modulo $\mathscr{F}$ of $K$ and denoted by $K^{s, \mathscr{F}}$.

For $v \in S(K) \backslash S$ let $\left(\frac{K^{s, s} / K}{v}\right)$ be the Frobenius automorphism of $v$ for $K^{s, *} / K$. Define an Artin symbol

$$
\left(\frac{K^{s, s} / K}{\bullet}\right): \mathscr{I}_{S}^{(*)} \longrightarrow \operatorname{Gal}\left(K^{s, *} / K\right)
$$

by the formula

$$
\left(\frac{K^{s, \mathscr{F}} / K}{\mathbf{c}}\right)=\prod_{v \in S(K) \backslash S}\left(\frac{K^{s, \mathscr{F}} / K}{v}\right)^{v(\mathbf{c})}
$$

Now I can formulate the main result of this paper.

Theorem. Let $K$ be a global field, $S \subset S(K)$ a finite set, $S \neq \varnothing$ in the function field case, $\mathscr{F}$ a cycle of $R_{S}$ and $K^{s, *}$ the $S$-ray class field modulo $\mathscr{F}$ of $K$.

i) $K^{s, s} / K$ is a finite abelian extension, unramified outside $S(\mathscr{F})$, and for $\alpha \in J_{K}^{(s)}$ we have

$$
\left(\alpha, K^{s, s} / K\right)=\left(\frac{K^{s, s} / K}{\iota_{s}(\alpha)}\right)
$$

ii) The Artin symbol induces an isomorphism

$$
\mathscr{I}_{S}^{(s)} / \mathscr{S}_{S}^{(\mathscr{s})} \stackrel{\longrightarrow}{\longrightarrow} \operatorname{Gal}\left(K^{s, \mathscr{F}} / K\right)
$$

iii) A place $v \in S(K)$ splits completely in $K^{s, *}$ if and only if either $v \in S$ or $P_{v, S} \in \mathscr{S}_{S}^{(\xi)}$.

iv) $K^{s,(1)}$ is the maximal unramified abelian extension of $K$ in which all prime $v \in S$ split completely.

v) Let $K$ be a function field, $F$ its field of constants and $F^{s, F}$ the 
field of constants of $K^{s, \mp}$; then

$$
\left[F^{s, \infty}: F\right]=\operatorname{gcd}\{\operatorname{deg}(v) \mid v \in S\} .
$$

Remarks. 1. The splitting property iii) in the above Theorem can be used to characterize $K^{s, s}$, cf. [2; "Excercise" 6].

2. Property iv) of the above Theorem has been used in [6] to define the generalize Hilbert class field; for $\mathscr{F}=(1)$ the above Theorem generalizes [6; Theorem 1.3].

3. In the number field case, $K^{\phi,}$ is the usual ray class field modulo $\mathscr{F}$ and $K^{\phi, \mathscr{F}}$ is the Hilbert class field of $K$.

Proof of the Theorem. i) By construction, $K^{s,} / K$ is abelian, unramified outside $S(\mathscr{F})$, and

$$
\left(\bullet, K^{s, \mathfrak{s}} / K\right): J_{K} \longrightarrow \operatorname{Gal}\left(K^{s, *} / K\right)
$$

has kernel $K^{\times} \cdot J_{K, S}^{g}$, so that

$$
\left[K^{s, *}: K\right]=\left(J_{K}: K^{\times} \cdot J_{K, S}^{\star}\right)<\infty .
$$

For $\alpha \in J_{K}^{(\Phi)}$ we have

$$
\begin{aligned}
\left(\alpha, K^{S, \mathscr{F}} \mid K\right) & =\prod_{v \in \mathcal{S}(K)}\left(\alpha_{v}, K_{v}^{S, \mathscr{F}} / K_{v}\right) \\
& =\prod_{v \in S(K) \backslash S}\left(\frac{K^{S, \mathscr{F}} / K}{v}\right)^{v\left(a_{v}\right)}=\left(\frac{K^{s, \mathscr{F}} / K}{\iota_{S}(\alpha)}\right),
\end{aligned}
$$

where $K_{v}^{s, *} / K_{v}$ denotes the local extension attached to $K^{s, *} / K$ at $v$.

ii) By definition of the $S$-ray class field, the Artin symbol $\left(\bullet, K^{s,} / K\right)$ induces an isomorphism

$$
J_{K} / K^{\times} \cdot J_{K, s}^{\mathscr{s}} \stackrel{\longrightarrow}{\longrightarrow} \operatorname{Gal}\left(K^{s, \Psi} / K\right),
$$

and by i) we have a commutative diagram

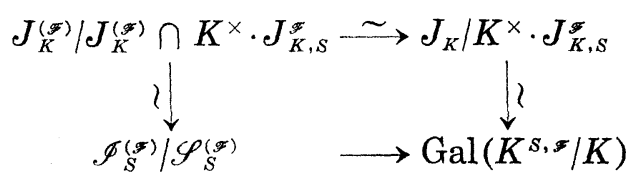

in which the bottom map is induced by the Artin symbol and the others are isomorphisms by Lemma 2.

iii) For $v \in S(K)$, define $e(v) \in J_{K}$ by

$$
e(v)_{v^{\prime}}= \begin{cases}1, & \text { if } v^{\prime} \neq v, \\ \pi_{v}, & \text { if } v^{\prime}=v,\end{cases}
$$


where $\pi_{v} \in K_{v}$ is an element with $v\left(\pi_{v}\right)=1$. Then $v$ splits completely in $K^{S, F}$ if and only if $e(v) \in K^{\times} \cdot J_{K, S}^{s}$; from this the assertion follows.

iv) Let $L / K$ be an unramified abelian extension of $K$ in which all places $v \in S$ split completely, and let $W \subset J_{K}$ be the open subgroup with $K^{\times} \subset W$ such that $L$ is class field to $W / K^{\times}$. Then $J_{K, S}^{(1)} \cdot K^{\times} \subset W$, and thus $L \subset K^{s,(1)}$.

v) Let $\bar{F}$ be an algebraic closure of $F$ and $\varphi$ the Frobenius automorphism of $\bar{F} / F$, so that $\operatorname{Gal}(\bar{F} / F)=\langle\bar{\varphi}\rangle$. If $\alpha \in J_{K}$, then

$$
(\alpha, K \bar{F} / K) \mid \bar{F}=\varphi^{\operatorname{deg}(\alpha)}
$$

with $\operatorname{deg}(\alpha)=\sum_{v \in S(K)} v\left(\alpha_{v}\right) \cdot \operatorname{deg}(v)$. Therefore, $F^{s, *}$ is the fixed field of $\left\{\varphi^{\operatorname{deg}(\alpha)} \mid \alpha \in K^{\times} \cdot J_{K, S}^{\mathcal{g}}\right\}$, and

$$
\left[F^{s, \mathscr{F}}: F\right]=\left(\mathbf{Z}: \operatorname{deg}\left(K^{\times} \cdot J_{K, S}^{\mathscr{F}}\right)\right)=\operatorname{gcd}\{\operatorname{deg}(v) \mid v \in S\} .
$$

From the above Theorem (esp. iii)) and Čebotarey's Density Theorem [ 3 ; ch. 5] it is easy to deduce a statement of Dirichlet's type on arithmetical progressions in holomorphy rings as follows.

Corollary. Let $R_{S}$ be a holomorphy ring in a global field, $\mathscr{F}$ a cycle of $R_{S}$ and $C \in \mathscr{J}_{S}^{(\mathscr{F})} / \mathscr{S}_{S}^{(\mathscr{F})}$ an $S$-ray class modulo $\mathscr{F}$. Then $C$ contains infinitely many prime ideals; more precisely, as $s \rightarrow 1+$,

$$
\sum_{\substack{P \in C \\ \text { prime ideals of } n_{S}}}\left(R_{S}: P\right)^{-s}=\frac{1}{\left(\mathscr{I}_{S}^{(\mathscr{F})}: \mathscr{S}_{S}^{(\mathscr{F})}\right)} \cdot \log \frac{1}{s-1}+O(1) .
$$

\section{LITERATURE}

[1] E. Artin, J. Tate, Class Field Theory, Benjamin, 1967.

[2] J. W. S. Cassels, A. Fröhlich (Eds.), Algebraic Number Theory, Academic Press, 1967.

[ 3 ] M. D. Fried, M. Jarden, Field Arithmetic, Springer 1986.

[4] S. Iyanaga, The Theory of Numbers, North-Holland, 1975.

[5] S. Lang, Algebraic Number Theory, Addison-Wesley, 1970.

[ 6 ] M. Rosen, The Hilbert class field in function fields, Expo. Math., 5 (1987), 365-378.

[7] M. Rosen, S-units and S-class group in algebraic function fields, J. of Algebra, 26 (1973), 98-108.

[ 8 ] A. Weil, Basic Number Theory, Springer, 1967.

[9] E. Weiss, Algebraic Number Theory, McGraw-Hill, 1963.

Institut für Mathematik,

Karl-Franzens-Universität,

Halbärthgasse $1 / I$,

A-8010 Graz, österreich. 